various properties of the system such as the mass of the atoms and the forces between them. If this frequency is inside a range of allowed frequencies called the phonon band (also determined by various properties of the crystal), the vibration can travel through the system, which means that heat is conducted.

We can imagine the atoms as a chain of particles that are connected to their neighbours by springs. Heat is conducted along the chain because vibrations travel from one particle to the next. When one end of the chain is attached to a hot material, and the other to something cooler, the particles at the hot end vibrate more and this motion travels along the chain to the cool end. If the particles in the chain are connected by ideal harmonic springs, they vibrate at the same frequency, regardless of their amplitude, so motion (heat) at one end of the chain is easily transmitted down the line, while the temperature of the chain remains uniform along its length.

In real chains, like DNA, the links between atoms are anharmonic rather than ideal so the vibration frequency also depends on amplitude or, equivalently, the temperature. More precisely, the frequency decreases as the amplitude of the vibrations increases, so heat is transmitted less efficiently.

We can take advantage of this dependence to control the flow of heat by devising a one-dimensional chain in which a strongly nonlinear segment with a temperature-dependent phonon band is sandwiched between two harmonic end segments, in which phonon bands do not vary with temperature ${ }^{2,3}$. Moreover, if the end segment on the right, say, has stiff springs that vibrate at a high frequency, whereas those on the left vibrate at a lower frequency, the three segments will have phonon bands at different frequencies. We can arrange these bands so that heat can flow from left to right, but not in the opposite direction (Fig. 1a). It is also possible to devise a thermal rectifier based on two nonlinear segments (Fig. 1b; ref 4).

The beautiful experiment of Zettl and co-workers possesses these features. They take nanotubes made of carbon and boron nitride, which are both quasi-onedimensional structures, and make them asymmetric by depositing trimethylcyclopentadienyl platinum in a non-uniform way along the length of the nanotube. Before this mass loading they do not observe any rectification when they measure the thermal conductance. After mass loading, however, all their measurements show that heat flows more easily from the high-mass end to the low-mass end than vice versa! This is the first experimental demonstration of a thermal rectifier.

Although the efficiency - defined as $\left[\left(K_{\mathrm{HL}}-K_{\mathrm{LH}}\right) / K_{\mathrm{LH}}\right] \times 100$, where $K_{\mathrm{HL}}$ is the thermal conductance when the heat flows from the high-mass to low-mass end - is small, around $2 \%$ for the carbon nanotubes and $7 \%$ for the best boron nitride nanotubes, we should remember that the performances of the first electronic diodes and transistors were very far from the efficiency of current devices. The authors speculate that solitons might be involved in the rectification process, but this is still to be confirmed.

This experiment, together with previous theoretical work, is only a preliminary step but it opens the way to applications such as cooling devices and heat-flow controllers for microscale chemical reactors in biotechnology. More generally, a thermal rectifier material could be used to cool materials and keep them cold. It is also possible that nature is already using this principle to control the flow of energy in biological molecules and cells, but a complete molecular dynamical study of a real biological molecule is needed to confirm if this is indeed the case. What is important for now, however, is that our ability to play with nonlinearities allows us to think about these possibilities.

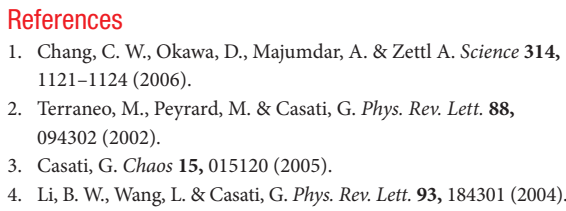

\title{
POLYMER STRUCTURES
}

\section{Getting to grips with complex liquids}

This might look like a gold-plated dome on an extravagant piece of architecture, but it is actually a droplet of liquid with an internal nanostructure. Most liquids form droplets with spherical caps if they cannot wet a surface, but other shapes - such as this terraced hyperbolic profile - are possible when the liquid possesses internal order or structure. Andrew Croll and colleagues at McMaster University in Canada and Reading University in the UK took this image of a diblock copolymer on a silicon surface with an atomic force microscope (Phys. Rev. Lett. 97, 204502; 2006). The layered structure is the result of interactions between the two different 'blocks' - polystyrene and PMMA - in the polymer.
However, the layers, which look like rings when viewed from above, disappear if the temperature goes above a certain value or if the molecular weight is increased. Croll and co-workers show that two competing interactions are responsible for the hyperbolic shape: edge tension drives liquid from the smaller disks near the top to the larger disks below, while edge repulsion prevents two adjacent edges from getting too close to each other. Understanding more about the behaviour of diblock copolymers should help researchers who are working to exploit the self-assembly properties of these materials in applications as diverse as nanoelectronics and synthetic muscle.

Peter Rodgers

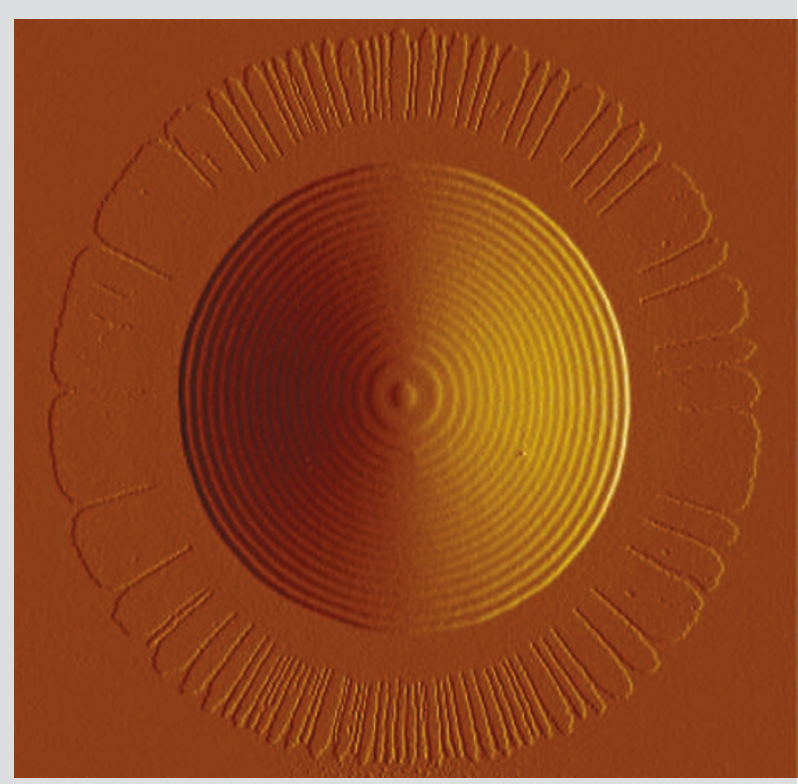

nature nanotechnology | VOL 2 | JANUARY 2007 | www.nature.com/naturenanotechnology 